\title{
INFLUENCIA DEL TIPO DE SECCIÓN TRANSVERSAL EN LA HIDRODINÁMICA DE LOS COLECTORES SOLARES DE LOS FOTOBIORREACTORES TUBULARES
}

\author{
INFLUENCE OF THE CROSS-SECTION TYPE \\ IN THE HYDRODYNAMICS OF SOLAR COLLECTORS \\ OF TUBULAR PHOTOBIOREACTORS
}

\author{
1 José Luis Ramírez Duque \\ 2 Mabel Angélica Ramos Lucumi
}

${ }^{1}$ Doctorado en Ciencias, Ingeniería Mecánica de Energía y Fluidos, Pontificia Universidad Javeriana, Cali- Colombia.

${ }^{2}$ Departamento de Ingeniería Mecánica, Universidad Autónoma de Occidente, Cali- Colombia

${ }^{1}$ Jose.ramirez@javerianacali.edu.co

2 maramos@uao.edu.co

\section{RESUMEN}

Diversos estudios sobre los cultivos de microalgas muestran la relación de la eficiencia fotosintética con la agitación. Esta incidencia fue evaluada en colectores solares para fotobiorreactores tubulares que poseen diversas geometrías en el perfil transversal (circular, octagonal, hexagonal y cuadrado). En este trabajo se realizó un estudio computacional de un cultivo de microalgas (flujo monofásico) para cada uno de estos colectores solares con un diámetro hidráulico de 2 in, 100 in de longitud y seis diferentes velocidades de entrada del cultivo al colector (de 0,25 a 0,5 m/s), para establecer la influencia de los perfiles en el comportamiento del fluido. Se evaluaron los parámetros de velocidad, caída de presión, flujo secundario y esfuerzo cortante; estableciendo que el colector con perfil hexagonal proporciona la mejor agitación debido a la irregularidad geométrica, aunque se deben utilizar velocidades de entrada del cultivo menores a $0.3 \mathrm{~m} / \mathrm{s}$ para garantizar un modelo de crecimiento continuo de las microalgas, de acuerdo con la tendencia de la bibliografía. Bajo este régimen de operación se encontró que los valores de esfuerzos cortantes no causan daños en la pared celular de las microalgas haciendo viable su implementación en plantas piloto.

Palabras clave: Agitación, Biomasa microalgal, Cultivos microalgales, Dinámica de fluidos computacional (CFD), Esfuerzos cortantes, Fotobiorreactor tubular (TFBR) 


\section{ABSTRACT}

Several studies in microalgae cultures show the relation between the photosynthetic efficiency and agitation, this effect was evaluated in solar collectors for tubular photobioreactors, which have different geometries in cross section (circular, octagonal, hexagonal and square). In this work, a computational study of microalgae culture (single-phase flow) for each of these solar collectors with a hydraulic diameter of 2 in, 100 in of length and six different culture inlet velocities to the collector $(0.25$ to $0.5 \mathrm{~m} / \mathrm{s})$ are performed to set the influence of profiles on the fluid behavior. The speed, pressure drop, secondary flow and shear stress was analyzed, establishing that the collector with hexagonal profile provides better agitation due to the irregular geometry, but the inlet velocities of the culture must be used in the collector for less than $0.3 \mathrm{~m} / \mathrm{s}$ to ensure a continuous growth model of microalgae according to the literature tendency. It was found, under this operation regime, that the shear stress values do not cause damage to the wall cell of the microalgae making this implementation feasible in pilot plants.

Keywords: Agitation, Computational fluid dynamics (CFD), Microalgae biomass, Microalgae cultures, Shear stress, Tubular photobioreactors.

\section{INTRODUCCIÓN}

El estudio de un fluido monofásico a través de un conducto es una de las combinaciones más elementales debido a que se establecen patrones comparativos para el análisis de otras configuraciones relacionadas, como el cambio de la presión, velocidad, viscosidad, numero de Reynolds, temperatura, etc. La posición del conducto, vertical u horizontal, es una variante delimitante para el análisis del desarrollo del flujo pues las propiedades de los fluidos cambian: en posición vertical, el movimiento del fluido es debido a la presencia de la fuerza gravitacional que está en función de la altura del ducto, mientras que en un ducto de posición horizontal el movimiento del fluido está relacionado con la caída de presión del flujo, lo que establece una correlación directa con la velocidad del flujo.

En el caso del colector solar de un fotobiorreactor tubular (FBR), el comportamiento del fluido que transita a través de él es similar al que si circulara por un ducto o tubería lisa. Anteriormente, García et al. (2009), Leeuwener et al. (2008) y Perner-Nochta et al. (1997), han diseñado colectores solares con agitadores estáticos para garantizar que todas las algas que se encuentran en el cultivo reciban la luz necesaria para realizar el proceso de fotosíntesis (Camacho et al., 1999 y Ugwu et al., 2005). Sin embargo, como una alternativa a este sistema de mezcla, en este trabajo propone la utilización de colectores solares no circulares que permitan ciertos niveles de turbulencia en el fluido, que podrían sustituir a los mezcladores del FBR.

Considerando que las microalgas presentan una flotabilidad neutra en el medio cultivo (Michels et al.,2010), en este artículo se analiza el comportamiento de un cultivo de microalgas como un fluido monofásico a través de los colectores solares horizontales de los fotobiorreactores tubulares de diferente perfil transversal (circular, octagonal, hexagonal y cuadrado). Se utiliza la herramienta de Dinámica de Fluidos Computacional (CFD) llamada Fluente, bajo el modelo estándar del esfuerzo de Reynolds. 


\section{FOTOBIORREACTORES}

Desde la década de los cincuenta, se han efectuado múltiples estudios científicos con el fin de obtener, a partir de las microalgas, una nueva fuente de energía capaz sustituir los hidrocarburos fósiles (principalmente el petróleo) y que, a su vez, proteja el medio ambiente. De hecho, gracias a estos microorganismos existen escenarios que están contribuyendo en gran medida a mejorar las condiciones atmosféricas terrestres, gracias a su capacidad fotosintética, ya que permiten reducir la fijación del carbono en la atmosfera causante del efecto invernadero, proporcionando un ambiente más seguro. El cultivo de estos microorganismos es de importancia mundial principalmente por sus derivados (farmacéuticos, nutracéuticos, agroquímicos, alimenticios, etc.). La biomasa que se obtiene a partir de las algas se utiliza para la producción de biocombustibles (biodiesel, etanol o metano), aditivos de alimentos y fertilizante orgánicos (Kim, 2015).

En caso de que se requiera la adaptación a condiciones medioambientales de todas las especies biológicas, se impacta sobre las condiciones de diseño y operación de los equipos (Eriksen, 2008). Por tal motivo, el uso de tecnologías foráneas llevaría a tener disminuciones en la eficiencia y producción de ácidos grasos, lo que hace imprescindible la implementación de tecnologías locales que tengan en cuenta todas las condiciones ambientales de la región.

Los fotobiorreactores (FBR) se caracterizan por garantizar las condiciones óptimas para que se realice el correcto crecimiento y reproducción de microalgas y cianobacterias, con inyección de nutrientes (tales como el dióxido de carbono, nitrógeno y fósforo), energía fotónica (la luz solar o iluminación artificial) y eliminación de desechos producidos por el proceso de la fotosíntesis, propia de este tipo de organismos (Chen et at., 2009). Entre los FBR más utilizados están los tubulares (tubular photobioreactor), columna de burbujas (bubble column photobioreactor) y panel o placa (flat panel photobioreactor) (Ramírez 2017).

Los FBR tubulares son sistemas cerrados desarrollados para la producción de microalgas que impiden que el cultivo tenga contacto con el aire, evitando cualquier contaminante externo al cultivo. Están compuestos por tubos de materiales transparentes (i.e. vidrio y policarbonato) rectos, enrollados o serpenteados, dispuestos en diferentes posiciones para capturar la radiación lumínica máxima para el cultivo de algas. El cultivo de microalgas en un FBR es un bache alimentado con un ciclo que va desde un almacenamiento o reserva (columna desgasificadora), hacia un colector solar y regresando al punto inicial de almacenamiento. En el colector solar se aplican varios métodos utilizados como generadores de movimiento para proveer la circulación del cultivo a través de él, por ejemplo los agitadores mecánicos y la inyección de aire (Camacho et al., 1999). Comúnmente, estos fotobiorreactores cuentan con una unidad Ilamada "Airlift" que, por medio de inyección de aire, genera el movimiento mientras que realiza la desgasificación en el colector, eliminando el oxígeno resultante de la fotosíntesis de las algas (Pruvost et al., 2008), también previene esfuerzos cortantes altos que producen daño a las células de las algas (Michels et al., 2010), como se muestra en la Figura 1.

La ventaja principal de los FBR es la gran área de exposición a la luz solar, por lo tanto, su actividad fotosintética es elevada al igual que su productividad de biomasa (Milledge y Heaven, 2013). Sin embargo, algunas desventajas son la poca transferencia de masa, altos niveles de $\mathrm{pH}$ y oxígeno en la mezcla y limpieza del FBR (Sánchez Mirón et al., 2000). 
Por medio de este trabajo se resalta la implementación de esta tecnología a las condiciones climáticas locales, con énfasis en el efecto de agitación que se puede lograr con los diferentes perfiles utilizados en los colectores solares de los fotobiorreactores tubulares. Aunque anteriormente se han realizado estudios sobre los flujos en ductos para aplicación ingenieriles como sistemas de tuberías hidráulicas relacionadas con la corrosión (Dai \& Shen, 2006) o en la dinámica de biofluidos para el flujo sanguíneo en el sistema vascular (Santamarina et al., 1998); no se han notificado estos estudios para los colectores solares de los fotobiorreactores tubulares.

\section{MODELO MATEMÁTICO}

Con el modelo de esfuerzo de Reynolds, RSM (Reynolds Stress Model) se pueden calcular las propiedades de los fluidos en cualquier punto, en un caso de flujo compresible o en el que haya transferencia de calor, además, es un prototipo detallado de turbulencia, como se señala en Alpma et al. (2005) y García et al. (2009). El RSM es usado para calcular el esfuerzo de Reynolds a partir de las ecuaciones diferenciales de transporte; la Ec. (1) representa el RSM para flujos con viscosidad constante (fluidos newtonianos)) (Alpma y Long, 2005).

$$
\begin{aligned}
& \frac{\partial}{\partial t}\left(\rho \overline{u_{i}^{\prime} u_{j}^{\prime}}\right)=\left.-\rho\left(\overline{u_{i}^{\prime} u_{k}^{\prime}} \frac{\partial u_{j}}{\partial u_{k}}+\overline{u_{j}^{\prime} u_{k}^{\prime}} \frac{\partial u_{i}}{\partial u_{k}}\right)-\frac{\partial}{\partial x_{k}}\left[\rho \overline{u_{i}^{\prime} u_{j}^{\prime} u_{k}^{\prime}}+\overline{p\left(\delta_{k j} u_{i}^{\prime}+\delta_{i k} u_{j}^{\prime}\right.}\right)\right] \\
&+\frac{\partial}{\partial x_{k}}\left[\mu \frac{\partial}{\partial x_{k}}\left(\overline{u_{i}^{\prime} u_{j}^{\prime}}\right)\right]+p\left(\overline{\left.\frac{\partial u_{i}^{\prime}}{\partial u_{j}}+\frac{\partial u_{j}^{\prime}}{\partial u_{i}}\right)}-2 \mu\left(\overline{\frac{\partial u_{i}^{\prime}}{\partial u_{k}}+\frac{\partial u_{j}^{\prime}}{\partial u_{k}}}\right)\right. \\
&\text { (2) } \left.\frac{\partial t}{\partial t} \overline{\left(\rho u_{i}^{\prime} u_{j}^{\prime}\right.}\right)=-P_{i j}-D_{i j}^{T}+D_{i j}^{L}+\phi_{i j}-\varepsilon_{i j}
\end{aligned}
$$

Siguiendo la Ec. (2), que utiliza la nomenclatura designada de la Ec. (1), el término a la izquierda de la ecuación representa la tasa de cambio de las componentes de velocidad, ui $u j$, a lo largo de las líneas de flujo; Pij (stress production) es la tasa de producción de $u i u j$ según el cortante medio y es uno de los generadores de turbulencia. El esfuerzo cortante se concibe por la interacción del esfuerzo transversal normal y deformación cortante; DTij y DLij son los términos difusión y representa la tasa de transporte espacial del $u i u j$, por la acción de las fluctuaciones turbulentas, las fluctuaciones de la presión; y la difusión molecular es el termino de presión-esfuerzo que representa la redistribución de la energía cinética turbulenta disponible entre los componentes de velocidad fluctuante, finalmente, $\varepsilon i j$ es la tasa de disipación de ui uj debido a la acción viscosa molecular (Hämäläinen, 2001).

En cuanto al esfuerzo cortante, éste es proporcional al módulo de la tasa de deformación ( $y$ ) y la viscosidad del fluido $(\mu)$, ver Ec. (3), (Wu y Shi, 2008).

$$
\text { (3) } \tau_{i j}=\mu \dot{\gamma}_{i j}
$$

Donde la tasa de deformación yij es el gradiente de velocidad del fluido en el colector característico para la velocidad de corte, (Vlaev et al., 2007), ver Ec. (4).

$$
\dot{\gamma}_{i j}=\frac{\partial u_{i}}{\partial x_{j}}+\frac{\partial u_{j}}{\partial x_{i}}
$$




\section{Dimensiones de los colectores solares}

El diámetro hidráulico y la longitud de estos conductos son los únicos factores que permanecen constantes para los diferentes perfiles, por lo que es necesario establecer las dimensiones generales de cada uno de los perfiles estudiados. Para el caso del conducto circular, el diámetro hidráulico corresponde al diámetro del perfil; pero para las otras configuraciones, el diámetro hidráulico está dado por la Ec. (5) (Aparecio y Cotta, 1990), donde se relaciona el área de la sección transversal $A$, con el perímetro mojado $p e$, del perfil del colector.

$$
D_{h}=4 \frac{A}{p e}(5)
$$

Bajo este criterio, se establecen las siguientes medidas para cada perfil, ver Figura 2:

Circular: $D=\mathbf{0 . 0 5 0 8} m$

Octágono: $L=\mathbf{0 . 0 2 1 0} \mathrm{m}$

Hexágono: $L=\mathbf{0 . 0 2 9 3} m$

Cuadrado: $L=\mathbf{0 . 0 5 0 8} m$

Determinación de la velocidad, caída de presión y flujo secundario en los colectores solares usando el método CFD

El cambio de presión en los diferentes conductos esta dado por el siguiente procedimiento:

\section{Generación de la malla}

Las ecuaciones del RSM son una representación matemática adecuada de los flujos, incluidos los turbulentos. Sin embargo, la resolución de estas ecuaciones para que se calcule el flujo turbulento requiere una discretización temporal y una malla de cálculo fina. Por esta razón, la generación de la malla es el paso inicial en las simulaciones CFD.
La cantidad de nodos en las mallas de estas geometrías oscilan entre un rango de 800.000 y 1.100.000. La malla tridimensional no estructurada utilizada para las secciones de los ductos es del tipo O-Grid. Este mallado fue realizado usando el software ICEM CFM (ANSYS 12.1), ver Figura 2. Los ductos de pared lisa considerados en este trabajo tienen un diámetro hidráulico, Dh, de 2" $(0,0508 \mathrm{~m})$ y una longitud, L, 100 veces mayor (5,08 m), se posicionaron en forma axial para permitir los efectos de entrada y salida.

\section{Dinámica de Fluidos Computacional|}

La simulación numérica del flujo monofásico (agua) en los ductos se realizó usando un software Fluent versión 12.1.2 (Ansys Inc.). El flujo laminar se analizó bajo un estado estacionario, con formulación implícita mediante el modelo de esfuerzos de Reynolds (presión-deformación lineal), usando diferentes velocidades de entrada del fluido en todos los conductos (ver Tabla 1) y una intensidad de turbulencia del $10 \%$. La condición en la entrada del conducto es de velocidad y en la salida es de presión igual a DP.

La turbulencia de los fluidos en estos colectores se ve significativamente afectada por las paredes, por lo que la dependencia de la malla en este análisis también radica en que el gradiente cerca de las paredes no sea muy grande, (Salim \& Cheah, 2009). Para tener éxito en la predicción del comportamiento del flujo en CFD, se enfatiza el parámetro YPLUS $(Y+)$, que tiende a disminuir a medida que la malla es más fina, y es la relación entre la influencia laminar y turbulenta en una celda. En este estudio, el máximo Y+ trabajado fue 0,45 (en el perfil hexagonal) lo que, según Gerasimov (2006), lo hace favorable para el modelado en la región cercana a la pared del colector solar ya que permite prevenir la sedimentación de 
las algas, lo que limitará la intensidad lumínica que requiere el cultivo (Provost et al., 2008).

Para garantizar la validez de los resultados obtenidos se deben controlar los parámetros que afectan a la resolución numérica del problema. Se puede actuar sobre el proceso iterativo definiendo los tipos de discretización, variando los factores de relajación y fijando los criterios de finalización de las iteraciones (Aparecio \& Cotta, 1990).

\section{RESULTADOS}

En este estudio de caso, el principal interés es el cambio de la velocidad, la caída de la presión a lo largo del ducto y el desarrollo del flujo secundario en función de la geometría. Esto se debe a la dependencia que poseen estos factores a las pérdidas producidas por la fricción del fluido en las paredes del ducto y al esfuerzo cortante que genera el fluido sobre las algas, que puede ocasionar daño celular. En las tablas 2, 3 y 4 se muestran las velocidades máximas alcanzadas por el fluido en el ducto y la presión a la entrada de los conductos (presión máxima), respectivamente. La aceptación de los resultados obtenidos puede ser examinada a través de diversos estudios numéricos o por comparación con los datos experimentales.

\section{Caída de presión}

El cambio, o la caída de la presión, en el conducto se deben, como ya fue mencionado, a las pérdidas por fricción en las paredes. La Figura 3 representa el cambio de la presión en los cuatro diferentes perfiles, usando una velocidad de entrada de $0,4 \mathrm{~m} / \mathrm{s}$.

Al linealizar las anteriores curvas, arrojan las siguientes ecuaciones:

Cuadrado: $\Delta P=-\mathbf{0 . 6 7 2} x+340.47$

Circulo: $\Delta P=\mathbf{- 0 . 6 4 8} x+328.14$
Hexágono: $\Delta P=-\mathbf{0 . 7 0 5} x+\mathbf{3 5 7 . 5 5}$

Octágono: $\Delta P=-\mathbf{0 . 6 8 4} x+\mathbf{3 4 6 . 4 2}$

Las pendientes de estas curvas demuestran las grandes pérdidas de presión que se presentan en el ducto hexagonal debido a la cantidad de aristas y al espacio que hay entre ellas, provocando una cavitación hidrodinámica en el cultivo que aumentaría su productividad en relación con el crecimiento celular de las microalgas (Gonzalez. y Muñoz, 2018)

\section{Velocidad}

La velocidad del flujo permite pronosticar el comportamiento del fluido en los conductos y establecer las situaciones críticas del proceso. En este estudio, el desarrollo de la velocidad se completó antes de los 0,5 m de la entrada del fluido en los ductos, lo que permite establecer las características propias del fluido; además, la máxima velocidad se presenta en el centro del ducto como se muestra en la Figura 4.

Los vectores de velocidad en el plano transversal de los ductos, despreciando los vectores axiales de velocidad, (ver Figura 5) describen el movimiento rotacional realizado por el fluido que da lugar al flujo secundario.

\section{Flujo secundario}

La resistencia causada por la fricción de las paredes de la tubería y la acción con la fuerza centrífuga combinadas produce una rotación en el movimiento del fluido conocida como flujo secundario; este movimiento es perpendicular al eje de la tubería y se interpone al movimiento axial del flujo (Belt et al.,2004; Molina Grima et al.,1999).

En la Figura 6 se muestra el flujo secundario para cada uno de los perfiles cuando la velocidad de entrada del agua es $0.4 \mathrm{~m} / \mathrm{s}$. El flujo secundario está relacionado a la agitación del 
fluido en el ducto debido a la rotación del flujo.

\section{Esfuerzo cortante}

Las fuerzas hidrodinámicas que se presentan en un colector del TFBR solar pueden causar altos niveles de esfuerzos cortantes que afectan las microalgas (Mazzuca et al., 2006; Sanchez et al., 2000), reduciendo su crecimiento a causa de la muerte celular. Sin embargo, para Michels et al. (2010), este movimiento de agitación en la mezcla es necesario para que las algas permanezcan en suspensión y para mejorar la distribución de luz. Así que, para optimizar el diseño del PBR, es importante conocer el máximo nivel de esfuerzo cortante que pueden soportar las microalgas.

La tolerancia al esfuerzo cortante depende específicamente de la especie de microalga; para Michels et al. (2010) la microalga Chaetoceros muelleri puede soportar 1,8 $\mathrm{Pa}$ sin presentar cambios en su proceso de crecimiento, mientras que para Mitsuhashi et al. (1995) la microalga Spirulina platensis también cambia su comportamiento cuando es afectada por un esfuerzo cortante mayor a 0,3 $\mathrm{Pa}$ pero no se producen daños celulares importantes.

En la tabla 5 se encuentran los esfuerzos cortantes máximos que logra alcanzar el flujo en cada uno de los conductos. Según Hämäläinen (2001), Wu \& Merchuk (2004) y Wu \& Shi (2008), los esfuerzos cortantes están relacionado directamente con la viscosidad del fluido y se presentan en mayor magnitud cerca de las paredes de los ductos. En la Figura 8 se ilustra este fenómeno en los colectores cuando la velocidad de entrada es $0,4 \mathrm{~m} / \mathrm{s}$.

\section{CONCLUSIÓN}

El flujo en el colector se puede calcular usando el modelo de esfuerzos de Reynolds, dado que este método considera la turbulencia del fluido. Tanto la presión como la velocidad están limitadas por la fricción que se genera entre el fluido y las paredes del ducto: A mayor cantidad de obstáculos, mayor es la pérdida de presión y la vorticidad del fluido, cuya agitación genera un esfuerzo cortante que afecta las células de las algas (Mazzuca et al., 2006; Michels et al., 2010; Mitsuhashi et al., 1995).

Con los resultados se permite establecer un parámetro de selección de ductos para transportar fluidos, como los cultivos de microalgas, en función de los movimientos axiales y rotacionales que se requieran y el cambio de la presión entre los extremos del conducto, siendo el perfil hexagonal el caso más crítico entre los ductos debido a la vorticidad que se produce por las esquinas del perfil. Este suceso es contrario a lo que sucede con el ducto circular, pues la fricción del fluido es mínima en las paredes.

Los valores arrojados por las simulaciones (ver Figura 6) demuestran que el perfil hexagonal es el que mejor comportamiento muestra en cuanto a flujo secundario $y$, por tanto, favorece la agitación del cultivo dentro del colector mejorando la actividad fotosintética (Gonzales y Muñoz, 2018). También se puede demostrar que los valores de esfuerzos cortantes, para los diferentes perfiles del colector (ver Tabla $5)$, son inferiores a los valores críticos que causan daños celulares en las microalgas (Mazzuca et al., 2006; Michels et al., 2010; Mitsuhashi et al., 1995; Vlaev et al., 2007); sin embargo, para controlar la tasa de crecimiento de las microalgas sugerida en la literatura, se debe trabajar con velocidades de entrada inferiores a $0,3 \mathrm{~m} / \mathrm{s}$ en el perfil hexagonal, teniendo en cuenta que este valor es dependiente de la especie de microalga utilizada.

Lamentablemente, la problemática que genera la fabricación de un colector solar en forma serpentín, como se ve en la Figura 1, y con 
perfil hexagonal es importante. Además, se debe limpiar periódicamente el colector para evitar que se dé una sedimentación del cultivo en las paredes del colector; este proceso en un colector de perfil hexagonal puede llegar a ser complejo debido a la dificultad en acceder a las aristas.

\section{AGRADECIMIENTOS}

A la Dirección de Investigaciones de la Universidad Autónoma de Occidente por la financiación del proyecto "Diseño de un prototipo de fotobiorreactor para la producción de biomasa a partir de algas como fuente de energía más limpia, en el departamento del Valle del Cauca.", aprobado con la resolución de rectoría No 6263.

\section{LITERATURA CITADA}

Alpma, E. \& Long, L.N. (2005). Separated turbulent flow simulations using a Reynolds stress model and unstructured meshes. In Processing of the 43rd Aerospace Sciences Meeting \& Exhibit (AIAA), Reno, USA, p. 1-14.

Aparecido, J.B. \& Cotta R.M. (1990). Laminar flow inside hexagonal ducts. Computational Mechanics 6, pp. 93-100.

Belt, R.J., Van't Westende, J.M.C., Portela, L.M., Mudde R.F. \& Oliemans, R.V.A. (2004). Particledriven secondary flow in turbulent horizontal pipe flows. In Processing of the 3rd International symposium on two-phase flow modeling and experimentation. Pisa, Italia.

Camacho Rubio,F., Acién Fernández, F.G., Sánchez Pérez, J.A., García Camacho, F. \& Molina Grima, E. (1999). Prediction of dissolved oxygen and carbon dioxide concentration profiles in tubular photobioreactors for microalgal culture. Biotechnology and Bioengineering 5(1), 71-86.

Chen, W.-y., Jiang, N., An, Y.-r., \& Yuan, Q.-h. (2009). Study on numerical simulation of singlephase injection device flow flied. In Processing of the Second International Conference on Information and Computing Science, (ICIC). Manchester, Inglaterra, p. 358-361.

Dai, Z. \& Shen, S. (2006). Effect of hydrodynamic factors on erosion-corrosion destroy and structure optimization of high pressure air cooler tubes. Journal of Pressure Equipment and Systems, 4: 37-41.

Eriksen, N.T. (2008). The technology of microalgal culturing. Biotechnol Lett 30, 1525-1536.

García, F. \& Haoulo, M. (2009). Estudio experimental de patrones de flujo bifásico aire-agua en tuberías horizontales y ligeramente inclinadas. Información Tecnológica 20 (3), 3-12.

García Camacho, F., Contreras Gómez, A., Acién Fernández, F.G., Fernández Sevilla, J. \& Molina Grima, E. (1999). Use of concentric-tube airlift photobioreactors for microalgal outdoor mass cultures. Enzyme and microbiol technology 24, 164-172.

Gerasimov, A. (2006) Modelling turbulent flows with FLUENT. Europa : ANSYS, Inc.

Gonzalez Fernandez, C. y Muñoz, R (2018). Microalgae-Based Biofuels and Bioproducts: From Feedstock Cultivation to End-products. Woodhead Publishing. 560.

Hämäläinen, V. (2001). Implementing an explicit algebraic Reynolds stress model into the threedimensional FINFLO flow solver. Helsinki University of technology, Laboratory of aerodynamics, Report No. B-52, Series B.

Kim, Se-Kwon (2015). Handbook of Marine Microalgae: Biotechnology advances. Academic Press

Leeuwner, M.J. \& Eksteen J.J. (2008). Computational fluid dynamic modeling of two phase flow in a hydrocyclone. The journal of the southern African institute of mining and metallurgy 106, 231-236

Mazzuca Sobczuk, T., Garcia Camacho, F., Molina Grima, E. \& Chisti, Y. (2006). Effects of agitation on the microalgae Phaeodactylum tricornutum and Porphyridium cruentum. Bioprocess Biosyst Eng 28, 243-250.

Michels, M., van der Goot, J.A., Norsker, N.H., Wijffels, R.H. (2010). Effects of shear stress on the microalgae Chaetoceros muelleri. Bioprocess Biosyst Eng 33, 921-927.

Milledge, J. J., Heaven, S. (2013). A review of the harvesting of micro-algae for biofuel production. Rev. Environ. Sci. Biotechnol. 12(2), 165-178.

Mitsuhashi, S., Hosaka, K., Tomonaga, E., Muramatsu, H. \& Tanishita, K. (1995). Effects of shear flow on photosynthesis in a dilute suspension of microalgae. Appl Microbiol Biotechnol 42, 744-749. 
Molina Grima, E., Acién Fernández, F.G., García Camacho, F. \& Chisti, Y. (1999). Photobioreactors: light regime, mass transfer, and scaleup. Journal of Biotechnology 70, 231-247.

Perner-Nochta, I., \& Posten, C. (2007). Simulations of light intensity variation in photobioreactors. Journal of Biotechnology 131, 276-285.

Pruvost, J., Cornet, J.-F. \& Legrand, J. (2008). Hydrodynamics influence on light conversion in photobioreactors: An energetically consistent analysis. Chemical Engineering Science 63, 3679-3694.

Ramírez Duque J. L. (2017). Viabilidad en la producción de biomasa microalgal a partir de fotobioreactores solares en el Valle del Cauca, Colombia. Revista de Investigación Agraria y Ambiental 8 (2), 127 - 140.

Sánchez Mirón, A., García Camacho, F., Contreras Gómez, A., Molina Grima, E. \& Chisti, Y. (2000). Bubble-column and airlift photobioreactors for algal culture. AIChE Journal 46 (9), 1872-1887.

Salim, S.M. \& Cheah, S.C. (2009). Wall Y+ strategy for dealing with wall-bounded turbulent flows. In Processing of the International MultiConference of Engineers and Computer Scientists (IMECS), Hong Kong, China.

Santamarina, A., Weydahl, E., Siegel, J.M. \& Moore, J.E. (1998) Computational analysis of flow in a curved tube model of the coronary arteries: effects of time-varying curvature. Annals of Biomedical Engineering, 26: 944-954

Ugwu, C.U., Ogbonna, J.C. \& Tanaka, H. (2005). Characterization of light utilization and biomass yields of Chlorella sorokiniana in inclined outdoor tubular photobioreactors equipped with static mixers. Process Biochemistry 40, 3406-3411.

Ugwu, C.U., Aoyagi, H., Uchiyama, H. (2008). Review: Photobioreactors for mass cultivations of algae. Process Biochemistry 99, 4021-4028.

Vlaev, S., Georgiev, D., Nikon, I. \& Elqotbi, M. (2007). The CFD approach for shear analysis of mixing reactor: verification and examples of use. Journal of Engineering Science and Technology 20 (2), 177-187.

Wu, X. \& Merchuk, J.C. (2004). Simulation of algae growth in a bench scale internal loop airlift reactor. Chemical Engineering Science 59, 2899-2912.

Wu, Z.Y. \& Shi, X.M. (2008) Rheological properties of Chlorella pyrenoidosa culture grown heterotrophically in a fermentor. J Appl Phycol 20, 279-282.

Yue, P., Dooley, J. \& Feng, J.J. (2008). A general criterion for viscoelastic secondary flow in pipes of noncircular cross section. Journal Rheol. 52 (1), 315-332.

\section{(9) $(1)(9$}


